\title{
MIGUEL FISAC Y EL HORMIGÓN COMO LENGUAJE ESTÉTICO
}

RAMÓN VICENTE DIAZ DEL CAMPO MARTIN-MANTERO 


\section{RAMÓN VICENTE DÍAZ DEL CAMPO MARTÍN-MANTERO}

Doctor por la Universidad de Castilla-La Mancha (España), donde desarrolla su actividad docente e investigadora. Sus principales líneas de investigación y publicaciones están relacionadas con el arte español durante la Guerra Civil española y la dictadura franquista. 


\section{RESUMEN \\ ABSTRACT}

Miguel Fisac Serna (Daimiel, 1913-Madrid, 2006) fue un singular arquitecto con una trayectoria profesional única que lo convirtió en uno de los artistas más particulares y conocidos del panorama español. Su carrera profesional se sitúa en la segunda mitad del siglo $X X$, siendo uno de los protagonistas del proceso de renovación de la arquitectura española que a partir de los años cincuenta buscó un camino de modernidad que dejara atrás los gustos academicistas de los años cuarenta. Su obra tiene un carácter muy personal que se destacó por una constante experimentación que le condujo a proponer numerosos avances técnicos con distintos materiales que fueron los protagonistas de una obra profundamente expresiva.

Palabras clave: Miguel Fisac, arquitectura contemporánea, siglo XX, materia, hormigón, modernidad, estética.

Miguel Fisac Serna (Daimiel, 1913 - Madrid, 2006), was a singular architect with extraordinary professional history that made him one of the best artists on the national scene. His work was in the second half of the twentieth century. He was one of the protagonists of the renewal process of the Spanish architecture from the fifties years. Fisac sought a way to leave behind modern forgetting academicians forms. Its buildings has a very personal appearance. He did numerous technical advances with different materials that were the protagonists of a work.

Keywords: Miguel Fisac, contemporary architecture, twentieth century, stuff, concrete, modernity, aesthetics. 
E n la actualidad existen múltiples sistemas de construcción basados en el uso del hormigón que ofrecen una gran variedad de formas, acabados y diseños. Las posibilidades estéticas y técnicas que ofrece el hormigón, especialmente en los cerramientos y cubiertas, son muy amplias, pero durante los años sesenta del pasado siglo la construcción con hormigón no se había desarrollado plenamente en España, y no se aprovechaban todas sus cualidades constructivas y estéticas. Miguel Fisac fue pionero en realizar indagaciones sobre él, y a partir de 1959 realizó una serie de investigaciones que fueron la seña de identidad de sus edificios. Preocupado por la expresión de los materiales, el hormigón era el que mejores posibilidades estéticas presentaba para el arquitecto. Realizó varios edificios donde su uso se convirtió no solo en un alarde técnico, sino que fue la piel de sus construcciones, dotándolas de una personalidad propia.

\section{EL ARQUITECTO INVENTOR}

El interés por la materia es uno de los puntos primordiales de la obra de Miguel Fisac, que ha llegado a ser definido por algunos autores como «arquitecto inventor» (Cortés, J. A., 1989) por la expresividad que adquirieron los materiales en sus construcciones. Fue consciente en todo momento de que la elección de unos determinados materiales influía de manera concluyente en el resultado final del edificio, sobre todo en cuanto a valores estructurales y estéticos. Tal era la importancia de los avances e indagaciones técnicas en su obra que fueron paralelos en todo momento a la evolución de sus lenguajes arquitectónicos. En la década del 50 patentó un ladrillo especial para cerramientos ligeros; posteriormente en los años sesenta creó distintas formas para cubrir espacios con hormigón, y luego aparecieron los denominados «encofrados flexibles» en los años setenta; todos estos procesos contribuyeron a manifestar la importancia que tuvieron para el arquitecto los materiales dentro de su proceso creativo.

El protagonismo de la materia en la concepción arquitectónica de un edificio estaba directamente relacionado con el programa de trabajo del arquitecto. Miguel Fisac publicó múltiples escritos a lo largo de su vida donde podemos observar que incide de forma constante en los aspectos metodológicos de la disciplina arquitectónica. El programa se basaba en la realización de un itinerario mental buscando soluciones constructivas a las necesidades del edificio (Arqués Soler, F., 1996, p. 37). Se establecía en las preguntas dónde, qué y cómo, que conducían a un interés por cuestiones sobre el lugar, la técnica y la funcionalidad. En primer lugar, Miguel Fisac organizaba espacialmente las necesidades de cada programa. Es decir, estudiaba para qué se construía el edificio, sintetizándolo gráficamente mediante organigramas (Besa Díaz, E., 2008). Una vez que había obtenido un esquema de los espacios necesarios era necesario analizar las circunstancias en las que se iba a insertar el edificio y estudiar el entorno del que formaba parte: ¿dónde?, elemento clave y verdadero protagonista de varias obras basadas en la arquitectura popular. En tercer lugar, con la información obtenida de los anteriores pasos, se planteaba con qué estructura y forma se llevaría a cabo, respondiendo así a la pregunta 
¿cómo?, definiendo de qué modo se debía materializar arquitectónicamente el proyecto. Por último, Fisac aplicó su sello personal, que él definió como «un no sé qué» (Fernández Cobián, E., 2005, p. 266). Esta singular forma de concebir el programa arquitectónico tuvo como resultado que sus obras no pudieran adscribirse a ningún lenguaje más que al del propio arquitecto. Debido a la importancia que adquieren las cuestiones relacionadas con el entorno y la materia en la concepción de sus proyectos podemos rastrear una serie de soluciones comunes que hacen que en Fisac veamos más que edificios una serie de construcciones con soluciones parecidas. Estos ejercicios harán que se tomen como punto de partida en algunas construcciones aspectos parciales de obras anteriores, asegurando una continuidad proyectual que se refleja tanto en aspectos técnicos como en espaciales, de tal manera que paulatinamente tienden a ir mejorando las soluciones técnicas de cada material según avance el tiempo, siempre que las condiciones económicas del proyecto lo permitan. Por lo tanto podemos entender o clasificar la obra de Miguel Fisac como la existencia de una serie de edificios construidos en distintos materiales, como el ladrillo, la arquitectura popular, el hormigón como cubierta y el hormigón como textura, etcétera (Fernández Cobián, E., 2005).

\section{LOS INICIOS, EL HORMICÓN COMO MATERIAL SECUNDARIO}

Los primeros años de ejercicio profesional de Miguel Fisac están directamente relacionados con la utilización del ladrillo. Realizó un gran número de proyectos con este material en los que se puede observar el interés del autor por conseguir una expresión acorde a cada uso y su función. La cerámica es un material muy versátil que ha sido empleado por los arquitectos en diversas formas y situaciones. Como material de construcción el ladrillo ha sido el más accesible y probablemente el más utilizado en la historia de la arquitectura española. Muchos autores a lo largo de sus trayectorias profesionales diseñaron elementos constructivos cerámicos que utilizaron como parte de sus lenguajes arquitectónicos. Miguel Fisac inició su carrera profesional a principios de los difíciles años cuarenta realizando varios edificios para el recién creado Consejo Superior de Investigaciones Científicas, en Madrid. Estos primeros edificios (Iglesia del Espíritu Santo y los institutos nacionales de Edafología y de Óptica) fueron considerados rabiosamente modernos en el panorama arquitectónico español, por su interesante intento de actualización del clasicismo, aunque una vez concluidos Fisac consideró que ese camino estaba agotado (Fisac, M., 1981). En ellos utilizó el ladrillo como material estructural en las bóvedas baídas de la nave principal de la Iglesia del Espíritu Santo; y en el Instituto de Óptica Daza de Valdés utilizó un ladrillo macizo de poco espesor.

El escenario cambió a finales de los años cuarenta, directamente vinculado con la situación política que vivía el país. España en el ecuador del siglo XX era un Estado que pretendía insertarse en el nuevo orden internacional, dejando atrás el aislamiento; las relaciones exteriores aumentaron progresivamente, tanto con países hispanoamericanos como con europeos, culminando en el año 1953 con los acuerdos de ayuda económica y mili- 


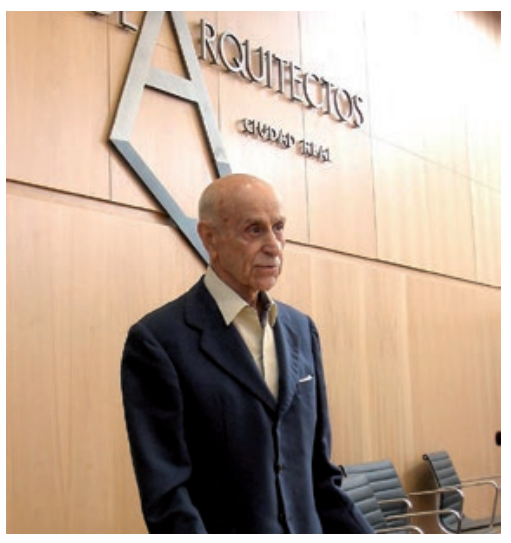

01.

tar entre España y Estados Unidos. Durante estos años un grupo de jóvenes arquitectos comenzó a desarrollar una arquitectura de tintes modernos directamente vinculada con los cambios de apertura que vivía el país. Por primera vez en mucho tiempo los autores españoles empezaron a hacer viajes al extranjero entrando en contacto con la realidad europea, al igual que llegaron a nuestro país algunas revistas extranjeras que mostraban una arquitectura totalmente desconocida para nuestros arquitectos (Urrutia, A., 1997). En este contexto Miguel Fisac hizo un viaje por distintas ciudades europeas en 1949, debido al proyecto de un centro de investigación para el que tuvo que visitar distintos laboratorios de estabulación de animales de experimentación (Delgado, E., 2014).

En algunos de estos edificios, como el Teologado de San Pedro Mártir o el Centro de Formación del Profesorado, ambos en Madrid, comenzó a utilizar, aunque de manera muy tímida, el material que definirá la siguiente serie: el hormigón. Su uso apareció en una fecha relativamente temprana en su obra, aunque será de una forma residual en comparación con el ladrillo. Podemos ver el inicio de una nueva época de experimentación en el estudio de las posibilidades del material, como ocurrió en la torre campanario del Teologado de los Dominicos, en Madrid, donde el
01.

\section{Miguel Fisac Serna (1913-2006).}

FUNDACIÓN MIGUEL FISAC

hormigón solo aparece empleado a gran escala en la torre, una gran estructura formada por 16 pilares cuadrados de 64 metros de altura que conjuga su función de campanario con la de imagen representativa del edificio (Fisac, M., 1960).

Otra solución en hormigón que convive con el ladrillo son los pórticos de membranas onduladas que se observan en el Centro de Formación del Profesorado, en Madrid (1953-55), y en el Colegio Apostólico de Arcas Reales (1952-53). En el primero de ellos el arquitecto utilizó unos moldes muy rudimentarios de cuerdas y escayola, consiguiendo unas marquesinas de contornos blandos para enlazar las distintas partes del proyecto, consiguiendo una interesante yuxtaposición de las soluciones en ladrillo y hormigón (Fisac, M., 1954). Estos elementos destacaron por su singularidad, ya que servían de comunicación entre los espacios abiertos y los cerrados de varios proyectos de la época, y tuvieron distintas variantes, intentando buscar mejorar el recurso, como podemos ver en los pórticos abiertos del Instituto de Enseñanza Media, de Málaga. Allí estas formas estructurales se convierten en el elemento formal que identifica al proyecto; sus pórticos de hormigón con pilares de sección variable establecen una continuidad y vinculan las distintas piezas del edificio (Loren Méndez, M. M., 2012). 
02

Pórticos del Colegio Apostólico

de los Padres Dominicos.

Valladolid. 1952.

FOTOGRAFÍA DEL AUTOR

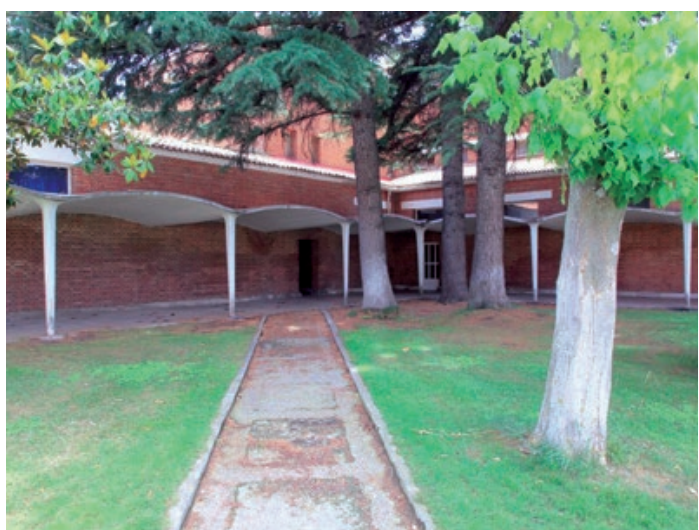

02.

\section{LENGUAJES DE HORMIGÓN}

Con la llegada de los años sesenta la obra de Miguel Fisac cambió, centrándose a partir de entonces en la utilización del hormigón como lenguaje estético. En esos años Miguel Fisac fue uno de los pioneros en la investigación de dicho material. Preocupado por la expresión de los materiales, el hormigón era el que mejores posibilidades estéticas presentaba para el arquitecto (Delgado Orusco, E., 2009). Realizó varios edificios donde este insumo se convirtió no solo en un alarde técnico sino que fue la piel de sus edificios, dotándolos de una personalidad propia. Fisac inició en esta etapa un camino individual en cuanto a su producción, destacando por su excesivo formalismo abstracto; sus edificios partían de una concepción de arquitectura adintelada y de las nuevas posibilidades que le brindaba la prefabricación; así comenzó a desarrollar una investigación con piezas prefabricadas de hormigón armado.

Los ensayos dieron como resultado una serie de piezas que él denominó «huesos» y que arrojaron unos interesantes resultados, siendo el reflejo del estado inicial en el que se encontraba la industria de prefabricación de elementos de construcción en España. Estos elementos responden a la necesidad que se planteó Miguel Fisac de buscar una solución técnica para poder reali- zar una cubierta usando el hormigón como material principal. Se buscaron distintas soluciones para salvar grandes luces usando para ello elementos prefabricados repetitivos.

El nombre de «huesos» viene de una curiosa anécdota protagonizada por el arquitecto. Tras haber realizado los cálculos de las piezas y dimensionarlas, el elemento resultante le recordaba algo, así que mandó a su mujer a la carnicería para que trajera distintos huesos de las columnas vertebrales de animales, y pudo ver cómo afinando al máximo los cálculos había llegado a un resultado que evocaba la solución por parte de la naturaleza en la evolución de la columna (Fisac, M., 1967, p. 9).

El origen de los «huesos» lo encontramos en las necesidades planteadas por el encargo del Centro de Estudios Hidrográficos, en Madrid (1960): para el laboratorio de hidráulica era necesario realizar una cubierta de 22 metros de luz (Fisac, M., 1964). Sin embargo, obras previas del arquitecto dan pistas sobre el nacimiento de la viga hueca. En el año 1958 Miguel Fisac realizó un interesante diseño de estructura adintelada para cubrir espacios de gran luz, se trató de un proyecto para el concurso de la Iglesia de San Esteban, en Cuenta (1960). El arquitecto planteó una solución para 
03.

PÁGINA OPUESTA

\section{Teologado de San Pedro Mártir. Madrid. 1954.}

FOTOGRAFÍA DEL AUTOR.

la estructura de cubierta formada por piezas prefabricadas de membrana de hormigón de doble curvatura, cuya sección evocaba lejanamente el perfil alado de una gaviota (Fisac, M., 1961).

Estas piezas forman parte de una búsqueda obsesiva por encontrar la pieza ideal, aquella que resolviera mejor todas las funciones constructivas, estructurales y arquitectónicas, en una solución única (González Blanco, F., 2010). En cada caso el arquitecto proyectaba la forma y disposición más adecuadas a la función -0 funciones- propiamente arquitectónica que pretendía resolver. Después decidía el tipo de tratamiento, en general de pretensado o postensado, tanto técnica como económicamente más conveniente, según fuera la luz, las cargas, el tamaño que habrían de tener las piezas, etcétera (Fisac, M., 1967). Fueron más de una docena las piezas diseñadas en su estudio, que fueron principalmente de cubiertas, aunque también ensayó algunos tipos de forjado y un tipo para fachada que utilizó en el edificio IBM, en Madrid (Fisac, M., 1970). Por su funcionamiento estructural se distinguen los «huesos» en dos grandes grupos: por un lado las vigas postensadas, basadas en dovelas y teselas, mientras que el segundo tipo corresponde a vigas continuas pretensadas que utilizaban tendones de acero de alta resistencia embebidos en el elemento estructural (González Blanco, F., 2006).

Esta solución fue perfeccionándose a lo largo de la década del 60 mediante las diferentes vigas, los «huesos» fueron evolucionando hasta conseguir una pieza compleja que no solamente permitió cubrir amplios espacios, ya que al mismo tiempo habilitaba la eva- cuación del agua de lluvia a través del canalón de su parte superior, poseían iluminación natural cenital y un parasol que evitaba la incidencia de luz directa (González Blanco, F., 2006). A lo largo de estos años Miguel Fisac generó más de una docena de piezas diferentes, de las cuales nueve fueron puestas en práctica, mientras que otras fueron proyectadas pero no utilizadas; incluso alguna fue usada experimentalmente para posteriormente ser desechada. Estas piezas se usaron en la mayoría de los edificios construidos por Miguel Fisac en los años sesenta, y se distribuyeron a lo largo de la geografía nacional: Madrid, Valladolid, La Coruña, Valencia, Vic y Jerez de la Frontera, entre otros lugares. El arquitecto patentó varias de estas soluciones en compañía de distintos colaboradores; cuando planteó estas soluciones, dentro del campo de la prefabricación contó con la experiencia de fabricantes que se convirtieron en sus habituales colaboradores (Fisac, M., 1964). Para las empresas a gran escala contó con la colaboración de Construcciones Barredo, verdadero pilar en el que se basa gran parte de las «vigas-hueso», que coincide con la experiencia paralela de Peiro SA, colaborando con Fisac en la edificación de proyectos de menor escala. Esta solución, tecnológicamente muy novedosa en España, se llegó a comercializar junto a Ricardo Barredo con el nombre de Huecosa SA, siendo uno de los pocos ejemplos de este tipo de experiencias en España hasta la fecha. La idea original era comercializar un sistema de prefabricación de piezas postensadas, en primer momento para cubrir las necesidades del Grupo Colomer, y posteriormente introducirse en el mercado (González Blanco, F., 2006). 


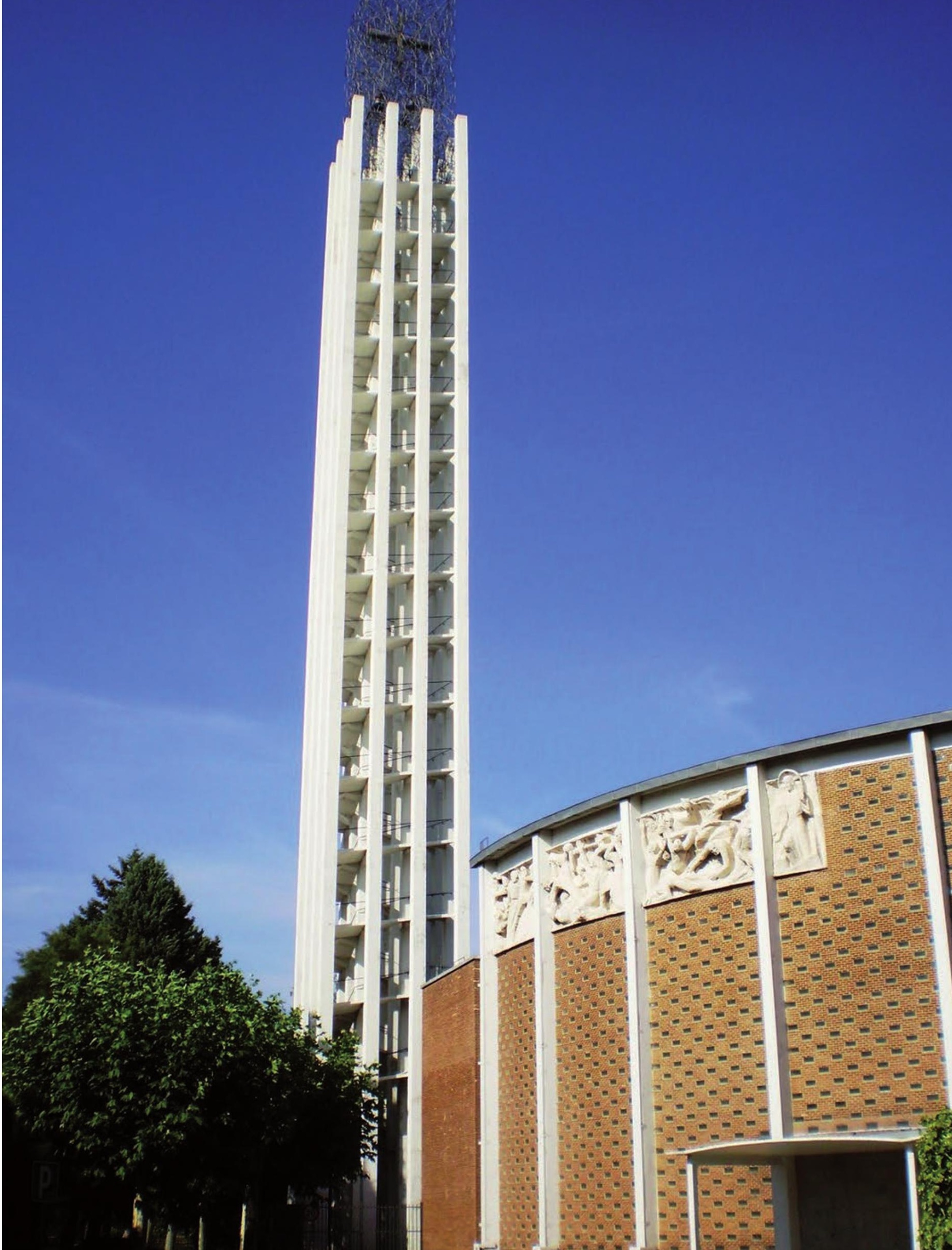




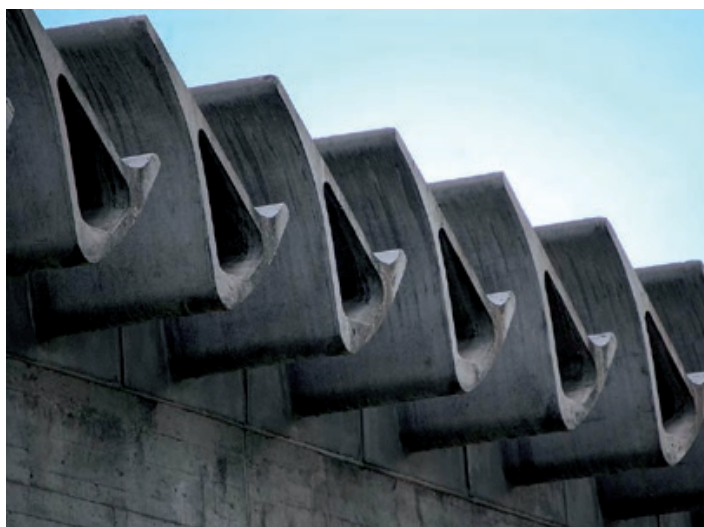

04

Para Miguel Fisac el hormigón armado fue un material no solo apto para la ejecución de formas estructurales del edificio, sino que también, como otros materiales, tenía unas características óptimas para la creación y delimitación de espacios. El arquitecto empezó a indagar en las características plásticas de los muros de hormigón en edificios donde experimentaba con el material como elemento de cubierta. Fisac estudió los distintos tipos de acabados de encofrados, para dejar el hormigón sin cubrir como elemento de las fachadas en sus obras (Álamo, M., 1997). En este sentido, el Centro de Estudios Hidrográficos (1960), de «gran sencillez formal y una absoluta expresividad estructural», según sus propias palabras, fue el edificio precursor del uso del hormigón como único material constructivo (Fisac, M., 1964, 21). En la Iglesia de Santa Ana de Moratalaz (1965) podemos ver el resultado de buena parte de todas las investigaciones sobre las posibilidades expresivas del hormigón aplicadas a un espacio religioso en pleno proceso posconciliar. Todo el conjunto se construyó con este material, dejando una lección verdaderamente magistral de originalidad.

\section{MUROS FLEXIBLES}

Con la llegada de los años setenta Miguel Fisac, que había vivido una gran popularidad durante los años anteriores, siendo considerado en la opinión popular como
04.

\section{Huesos del Centro de Estudios Hidrográficos. Madrid. 1960.}

FOTOGRAFÍA DEL AUTOR

uno de los arquitectos modernos por antonomasia, se enfrentó a una situación de falta de encargos y de un cierto olvido social. Debido al descenso de proyectos, en el año 1977 cerró su estudio en Madrid y decidió trasladarse durante largas temporadas a La Mancha. Durante estos años realizó algunos encargos esporádicos, principalmente obras en la provincia de Ciudad Real, y algunas intervenciones en obras suyas anteriores. Es muy interesante una serie de intervenciones en el patrimonio histórico de Castilla-La Mancha que el arquitecto llevó a cabo durante su destierro manchego, como la rehabilitación del Teatro Municipal y el campanario del Convento de la Encarnación, en Almagro, y el Castillo de Calatrava la Nueva, entre otras.

La falta de trabajo hizo que el artista centrara buena parte de su tiempo en su faceta de colaborador para distintos periódicos y publicaciones, contrastando con un menor número de apariciones y referencias al arquitecto manchego en las revistas especializadas en arquitectura. Su firma aparece frecuentemente en $A B C$ y Blanco y Negro, donde el arquitecto plasmó sus inquietudes, pensamientos, reflexiones y denuncias, contribuyendo estas últimas a crear una imagen polémica del artista, perfectamente resumida por Francisco Umbral, que lo definió como «el viejo arquitecto Miguel Fisac tiene algo de intelectual cabreado y algo de al- 
05.

Interior de la Iglesia de Santa Ana.

Madrid. 1965.

FOTOGRAFÍA DEL AUTOR

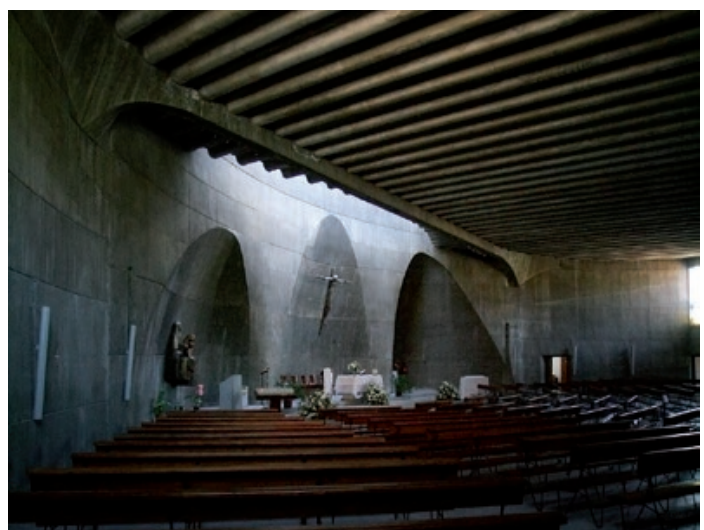

05.

deano manchego» (Umbral, F., 1994, p. 21). La pintura fue otro de los campos en los que se volcó en su tiempo libre (De Roda Lamsfus, P., 2007). La experimentación con la materia también se dejó ver claramente en esta faceta, ya que realizó un importante número de obras con una técnica en la que mezclaba tierras y látex que aplicaba directamente al lienzo puesto en posición horizontal. La mayoría de ellas son temas referentes a la arquitectura, con una paleta donde abundan los tonos pardos y ocres obtenidos de las arenas del entorno de La Mancha.

En el ámbito arquitectónico, la última etapa profesional del arquitecto fue al mismo tiempo la más incomprendida por parte de sus compañeros arquitectos y la crítica especializada. En ella se centró en nuevas investigaciones sobre las posibilidades del hormigón que no tuvo apenas respuesta entre sus colegas, y Miguel Fisac se vio relegado a un periodo de olvido profesional, más significativo aun si tenemos en cuenta que se produjo después de tres décadas de gran éxito. Indagó sobre la característica que se podría distinguir como exclusiva y característica del hormigón, y la encontró en su original estado pastoso. El arquitecto buscó la manera de conseguir un tipo de molde que dejara constancia de que aquella masa había sido en algún momento blanda, para darle al hormigón una expresividad nueva y propia. nó como encofrados flexibles, utilizando para ellos un nuevo sistema de moldes en el proceso del encofrado.

El sistema de encofrados flexibles para hormigón fue patentado a principios de la década del 70 (Fisac, M. 1972). El invento consistió en la formación de encofrados mediante unas estructuras que daban al material un aspecto totalmente nuevo al utilizado tradicionalmente. En esos esqueletos para el encofrado se situaba una lámina de un material flexible, principalmente plásticos o similares, que configuraba distintos huecos y formas. Sobre ese molde se vertía el hormigón, lo que permitía moldear las superficies y obtener un paramento que reproducía la configuración que tenía la estructura del encofrado, intentando plasmar en el resultado final la huella genética de su estado pastoso original.

Esta indagación sobre las posibilidades de los materiales desarrolló un nuevo lenguaje de la forma arquitectónica a través de un método de construcción simple e innovador que cambiaba drásticamente la visión tradicional del hormigón armado en las obras, y aparecieron nuevas oportunidades para el diseño arquitectónico. Con la nueva técnica de muros flácidos el arquitecto construyó algunos edificios, como el hotel Tres Islas,
A partir de entonces experimentó con lo que denomi- 


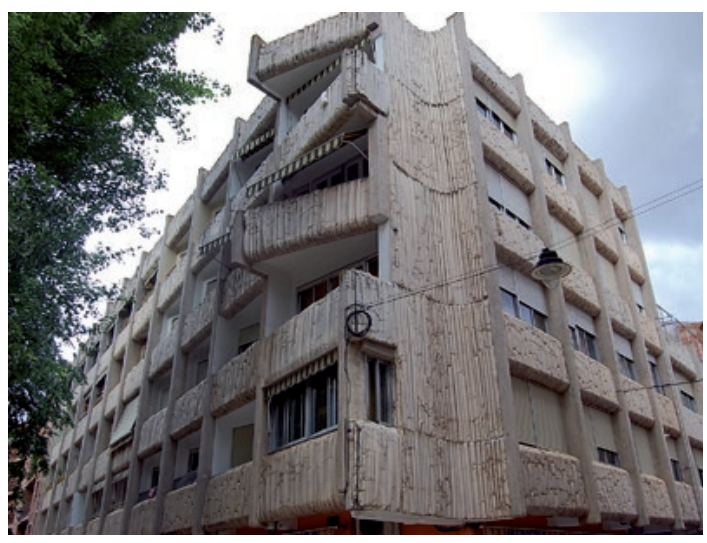

06.

en Fuerteventura, el Mausoleo de Félix Rodríguez de la Fuente o en la Parroquia de Nuestra Señora de Altamira. Este peculiar sistema fue aplicado también en la construcción de su propio estudio, situado a las afueras de Madrid, y en su casa particular en Almagro; en estos lugares se recluyó durante una prolongada temporada de marginación y se convirtieron en campo de pruebas para nuevos experimentos. Por ejemplo, en su estudio del Cerro del Aire realizó varias pruebas in situ de estos ensayos de hormigón flexible en muros verticales sin moldes previos, aunque la solución resultó cara y no totalmente satisfactoria (Arqués Soler, F., 1996). Fisac mostró a partir de entonces su interés por la prefabricación como solución óptima para estos elementos, aunque hubo algunos proyectos donde se realizaron a pie de obra personalmente con buenos resultados, como fue el caso de su propia vivienda o de un pequeño encofrado en la ermita de San Pedro, en Almagro, donde el arquitecto presumía de ejecutar no solo in situ sino personalmente. Fruto de estas convicciones surge la patente de 1983, titulada «Sistema de fabricación de elementos de fachada para la construcción» (Fisac, M., 1983). Fue un sistema de prefabricación de los encofrados flexibles ya patentados con anterioridad, pero en este caso especificando claramente su fabricación como elementos prefabricados.
06.

Edificio de viviendas Daimiel (Ciudad Real). 1976.

FOTOGRAFía DEL AUTOR

07.

Viviendas de Miguel Fisac en Almagro (Ciudad Real). 1978.

FUNDACIÓN MIGUEL FISAC

08.

Edificio de oficinas en San Juan (Alicante). 1988.

FOTOGRAFIA DEL AUTOR

Con este procedimiento, la cara vista del hormigón una vez fraguado presentaba un aspecto diferente al resultante de los sistemas convencionales de encofrados, mostrando unos abombamientos, pliegues, arrugas e irregularidades que ofrecían un efecto desconocido hasta el momento, con aspectos decorativos y ornamentales totalmente originales y con una ilimitada combinación de formas y dibujos. Además para su elaboración no era necesaria una mano de obra calificada, lo cual, unido a los bajos costes de los elementos que formaban los moldes, hicieron de esa patente una forma de construir muy original y accesible.

\section{A MODO DE CONCLUSIÓN}

En la obra de Miguel Fisac la materia se convirtió en un elemento de gran importancia para el diseño de sus proyectos. Sus indagaciones e inventos sobre distintos materiales fueron constantes a lo largo de su trayectoria profesional, y en paralelo con la evolución de sus lenguajes arquitectónicos. Su primera etapa estuvo marcada por el ladrillo y la arquitectura popular manchega, dando paso a partir de los años sesenta a una fructífera indagación sobre el hormigón. Hasta esa fecha pocos arquitectos habían sabido sacarle tanta expresividad a ese material, con la excepción de Félix Candela, que 


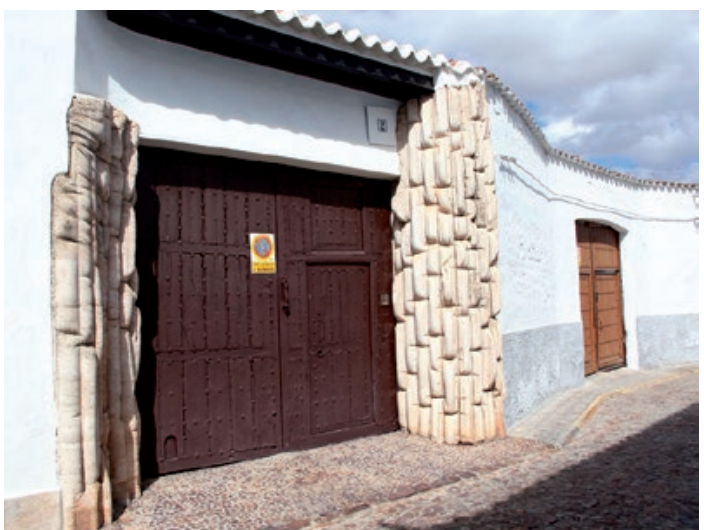

07.

en el año 1951 ya utilizó tela de saco sobre perfiles de carpintería para construir estructuras. Las indagaciones de Fisac distan mucho de algunas investigaciones arquitectónicas que en los últimos años, y con una base tecnológica, han dado resultados semejantes. Este interés por la investigación sobre materiales estuvo presente hasta el final de sus días. Miguel Fisac falleció en 2006 sin llegar a ver ejecutada su última patente, denominada «arquitectura vertida», que se estaba llevando a cabo en un edificio de viviendas en Vallecas, fruto de décadas de investigación sobre los problemas de la construcción de viviendas. Desde sus comienzos en la profesión, Miguel Fisac se interesó de una manera

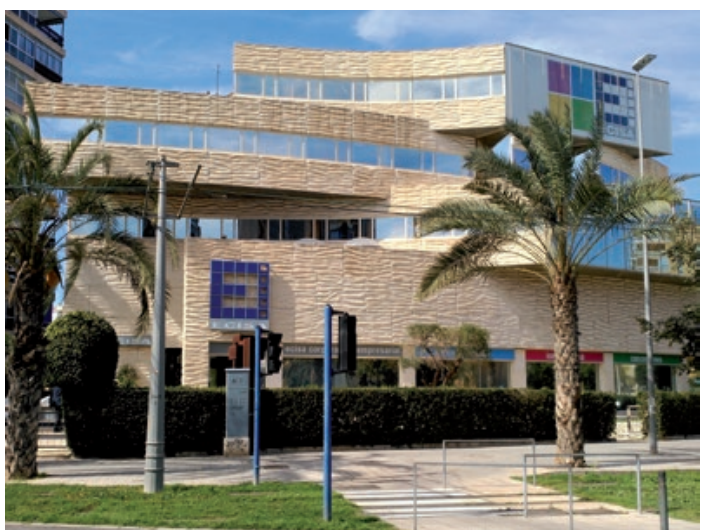

08.

especial por el tema de la vivienda social, obteniendo en 1950 el primer premio en un concurso convocado por el Colegio de Arquitectos de Madrid. Su sistema tiene su origen en dos patentes de prefabricación integral de 1965 y 1969 para edificios de viviendas que no llegaron nunca a ponerse en práctica (González Blanco, F., 2012). En su última patente el arquitecto creó un método de construcción que permitía realizar una serie de paneles prefabricados huecos con todas las instalaciones necesarias incluidas (electricidad, fontanería, desagües, calefacción, etcétera), que una vez que llegaban a la obra solo tenían que ser montados y rellenados con hormigón líquido. . 


\section{BIBLIOGRAFIA}

ÁLAMO, M. (1997). «Vértebras de hormigón», en Revista de los Ministerios de Fomento y Medio Ambiente, 453, pp. 81-84.

ARQUÉS SOLER, F. (1996). Miguel Fisac. Madrid: Pronaos.

BESA DÍAZ, E. (2008). «Miguel Fisac, una metodología proyectual», en Espacio, Tiempo y Forma, 20-21, pp. 391-415.

CORTÉS, J. A, (1989). «Miguel Fisac, arquitecto inventor», en BAU, 1, pp. 77-101.

DE RODA LAMSFUS, P. (2007). Miguel Fisac. Apuntes y viajes. Madrid: Scriptum.

DELGADO ORUSCO, E. (2009). «Las Iglesias de Miguel Fisac», en Fernández Cobián, E. Arquitecturas de lo sagrado. Memoria y proyecto (pp. 130-161). La Coruña: Netbiblo.

DELGADO, E. (2014). «Sota y yo. Entrevista a Miguel Fisac en el Cerro del Aire», en Boletín Académico. Revista de investigación y arquitectura contemporánea, 4, pp. 83-90.

FERNÁNDEZ COBIÁN, E. (2005). El espacio sagrado en la arquitectura española contemporánea. Santiago de Compostela: COAG.

FISAC, M. (1954). «Iglesia en Valladolid», en Informes de la Construcción, 66, pp. 148-149.

- (1954). «Más sobre casas en cadena», en Revista Nacional de Arquitectura, 148, pp. 14-16.

- (1960). «Teologado de San Pedro Mártir para los padres dominicos en Madrid», en Informes de la Construcción, 118, pp. 148-157.

- (1961). «Anteproyecto de iglesia parroquial de San Esteban, Cuenca», en Arquitectura, 25, pp. 13-51.

- (1964). «Centro de Estudios Hidrográficos», en Informes de la Construcción, 157, pp. 21-30.
- (1967). «Breves reflexiones de Miguel Fisac», en Arquitectura, 99, pp. 9-10.

- (1970). «Edificio de oficinas IBM, en el Paseo de la Castellana, 4. Madrid», en Cuadernos de Arquitectura, 78, p. 89.

- (1972). Memoria descriptiva: "Sistema de encofrados flexibles para hormigón», memoria de patente ES0382096. Oficina Española de Patentes y Marcas.

- (1981). «Asplund en el recuerdo», en Quaderns d'Arquitectura i urbanismo, 147, pp. 32-33.

- (1983). Memoria descriptiva: «Sistema de fabricación de elementos de fachada para la construcción», memoria de patente ES8600112 A1. Oficina Española de Patentes y Marcas.

GONZÁLEZ BLANCO, F. (2006). «Razón y ser de los tipos», en Informes de la Construcción, 503, pp. 41-48.

- (2010). Los huesos de Fisac. La búsqueda de la pieza ideal (tesis doctoral). Madrid: Universidad Politécnica de Madrid.

- (2012). «Gz/10. Un prototipo experimental de vivienda unifamiliar. Aplicación práctica de la última patente del arquitecto Miguel Fisac», en Informes de la Construcción, 526, pp. 153-166.

LOREN MÉNDEZ, M. M. (2012). «Tecnología, materia y lugar: procesos de modernización en la obra española de postguerra. Instituto de Enseñanza Media, Málaga», en Informe de la Construcción, 526, pp. 167-177.

UMBRAL, F. (21 de noviembre de 1994). «Fisac», en El Mundo, 21.

URRUTIA, A. (1997). Historia de la arquitectura española. Madrid: Cátedra. 